\title{
Signs and symptoms of genetic conditions - A handbook
}

\author{
Joann N. Bodurtha ${ }^{\mathrm{a}, \mathrm{b}, *}$ and Jessica Duis ${ }^{\mathrm{a}, \mathrm{b}}$ \\ ${ }^{a}$ Department of Pediatrics, Johns Hopkins, Baltimore, MD, USA \\ ${ }^{\mathrm{b}}$ McKusick-Nathans Institute of Genetic Medicine, Baltimore, MD, USA
}

Received 5 December 2014

Revised 5 December 2014

The book entitled "Signs and Symptoms of Genetic Conditions - A Handbook" by Louanne Hudgins, Helga V. Toriello, Gregory M. Enns, and H. Eugene Hoyme. Oxford, Oxford University Press, 2014, ISBN 978-0-19-993097-5, first edition.

The learning curve in the field of genetics is steep. There is so much to learn that knowing where to even begin can be overwhelming. My first goal upon entering the field was to find was a good text to guide me. In the months before starting my fellowship, I asked everyone for advice on what to purchase and ended up with a small collection of books - all of which were not quite as easy to digest as I had hoped. I picked up Signs and Symptoms of Genetic Conditions with the trepidation that I might not retain much, and was pleasantly surprised that the information was both easily understood and retained.

The authors dive right into genetics with some descriptions of the lab tests often ordered by geneticists. It is quite straightforward, with many nuggets

\footnotetext{
*Corresponding author: Joann N. Bodurtha, MD, MPH, FAAP, FACMG, Professor of Pediatrics and Oncology, Department of Pediatrics, Johns Hopkins, McKusick-Nathans Institute of Genetic Medicine, 600N. Wolfe St., Blalock 1012, Baltimore, MD 21287, USA. Tel.: +1 410955 1699; Fax: +1 410614 9246; E-mail: jbodurt1@jhmi.edu.
}

important for managing patients with genetic conditions. For example, the description of the esterified fraction of carnitine was extremely helpful in understanding the importance of stress dosing levocarnitine in patients with certain inborn errors of metabolism. The chapters continue by addressing specific diagnoses and discussing considerations from history and physical exams to honing in briefly on genetic differentials. This is useful when faced with a specific question during a genetics consultation or when seeing a referral in clinic to address specific concerns such as short stature. General pediatricians considering further diagnostic evaluation and possible referral to a geneticist would also find this book invaluable.

Common themes of the different chapters are the accessibility of language and the focus on broad strokes with key genetic diagnoses highlighted in table format. These traits make this book an excellent reference for residents entering into the field of genetics or general pediatricians starting the work-up for patients in consideration of genetic diagnoses. The tables provide a quick reference that can be utilized repeatedly. However, attention is needed due to some minor mistakes in the content. For example, the table that addresses mid-parental height switches the calculation for boys and girls A strength of this book is the list of references 
included at the end of each chapter that allows for one to read more in-depth about the topic addressed in that chapter.

I highly recommend this book be provided to newly starting residents and budding future geneticists interested in the field of metabolism or dysmorphology. It can be read in full to give a brief overview of the questions often asked when a geneticist consults on a patient, or it can utilized to search the contents to answer a specific question.

In summary, this handbook is an important contribution to the available genetics texts as it covers a broad range of topics from short stature to hyperammonemia in an accessible manner ideal for beginners in the field. The chapters focus on key syndromes and diagnoses that should be considered when faced with a particular sign or symptom. With the expanding knowledge in genetics, this handbook reminds physicians of the building blocks and provides them with the tools to start an appropriate work-up and discuss an appropriate differential.

As a teaching attending in pediatrics and genetics, I share this book with residents and medical students on rotation with us and have gotten universally good feedback about the utility of the thirty-one chapters. Most are under twenty pages, and enhanced by black and white photos, multiple tables and algorithms and can be read in less than 30 minutes. A reasonable balance is struck between the mention of specific genes and categories, with somewhat more emphasis on pediatric presentations than adult conditions. I recommend this book to geneticists, genetic counselors, and pediatricians for efficient overviews focused on the clinical assessment and diagnostic evaluation of key real world presentations of patients 